\section{ORIGINAL RESEARCH}

M. Ghali Eskander

A. Leung

D. Lee

\title{
Style and Content of CT and MR Imaging Lumbar Spine Reports: Radiologist and Clinician Preferences
}

BACKGROUND AND PURPOSE: Several studies have examined clinician preferences regarding the style of body sonography and CT reports. Our study is the first to examine clinicians' and radiologists' preferences in lumbar spine CT and MR imaging reports with respect to content and format and specific components such as management suggestions by the radiologist.

MATERIALS AND METHODS: A spine report survey, which consisted of 3 case scenarios, each with 6 different reports varying in content and format, was mailed to clinicians and radiologists. Their preferences regarding content, format, and management suggestions were gathered.

RESULTS: A total of 89 clinicians (49\%) and 31 radiologists (53\%) responded. Both clinicians and radiologists preferred reports with moderate or detailed instead of limited content $(P<.01)$. Itemized and prose formats were equally acceptable to clinicians and radiologists. Although both groups identified moderate CT technique description as ideal, more clinicians valued the inclusion of the quality of a CT study $(P<.001)$. Specialists preferred reports with greater detail but no recommendations, whereas family physicians preferred less detail but wanted specific management suggestions $(P<.01)$. Neuroradiologists $(75 \%-100 \%)$ were more likely to provide management suggestions than non-neuroradiologists (23\%-59\%).

ConcLusıons: Clinicians favored lumbar spine CT and MR imaging reports with detailed content in either itemized or structured prose formats, irrespective of the modality or the extent of abnormalities reported. Family physicians preferred management suggestions from the radiologists. Specialists, however, preferred a review of the radiologic findings and an opinion without specific recommendations. To optimize patient care, radiologists should be mindful of these preferences and consider tailoring their reports to their audiences.

ABBREVIATIONS: BIRADS = Breast Imaging Reporting and Data System; FP = family physician; $\mathrm{N}=$ neurological specialist; $\mathrm{N} / \mathrm{A}=$ the question was not asked in the study; $\mathrm{NN}=$ non-neurological specialist; $\mathrm{MRI}=\mathrm{MR}$ imaging; $\mathrm{PMR}=$ physical medicine and rehabilitation physicians

$\mathbf{E}_{\mathrm{n} \text { ex }}^{\mathrm{n}}$ ffective communication is a critical component of diagnostic imaging if quality patient care is to be achieved. ${ }^{1}$ Clinicians request imaging studies, interpret the subsequent reports issued by the radiologists, and then act on the findings and opinions in the report. Therefore, to achieve effective communication, the radiology report should be tailored to meet the needs of the clinicians in terms of format, content, and recommendations. However, radiologists typically have unique and individual reporting styles that often do not meet the needs of their clinicians.

Although multiple studies have attempted to define the ideal report, the recommendations from the American College of Radiology are simply that a radiology report should include patient demographics, relevant clinical information, procedures and materials, findings, potential limitations, clinical issues pertaining to the initial imaging request, compari-

Received March 9, 2010; accepted after revision May 12

From the Department of Medical Imaging, London Health Sciences Centre, London, Ontario, Canada.

Paper previously presented at: Annual Meeting of American Society of Neuroradiology and the Neuroradiology Education and Research Foundation, May 15-20, 2010; Boston, Massachusetts; and American Roentgen Ray Society, May 2-7, 2010; San Diego, California.

Please address correspondence to Andrew Leung, MD, Department of Medical Imaging, London Health Sciences Centre, 339 Windermere Rd, London, ON, Canada, N6H 5H6; e-mail: andrew.leung@|hsc.on.ca

indicates article with supplemental on-line table and appendix.

DOI 10.3174/ajnr.A2218 son with previous studies and reports, as well as a final diagnosis, differential diagnosis, and follow-up suggestions. ${ }^{1}$ It has been suggested that a more structured report organization and standardized radiologic lexicon has the potential to improve patient care, data mining, audits, peer review, outcome assessments, and medical education across the medical specialties. ${ }^{2}$ BIRADS has been successfully used in breast imaging for more than a decade. While CT and MR imaging have revolutionized the level of detail revealed by imaging in the work-up of degenerative conditions of the lumbar spine, there is no BIRADS-equivalent reporting structure in spine imaging.

Consequently, spine reports vary widely in their level of detail. If the required information is present in the report but presented with either too much or too little description, the report may be insufficient to meet the needs of the clinicians. Also, the value of the report will be reduced if useful management suggestions regarding further work-up or treatment are absent.

Our study aimed to assess style preferences in terms of content and format among clinicians and radiologists in an attempt to improve the utility of CT and MR imaging spine reports.

\section{Materials and Methods}

\section{Subject Selection}

The study design was based on a physician survey model. ${ }^{3}$ A referring physician data base was sorted to yield a list of referring physicians to 
Table 1: Number of respondents from each specialty surveyed, their specialty averaged years of experience, and utility/volume of studies ordered/reported

\begin{tabular}{lccc}
\hline Specialty & $\begin{array}{c}\text { Respondents/ } \\
\text { Surveyed }\end{array}$ & Average Years of Experience & $\begin{array}{c}\text { Average No. of Studies } \\
\text { Ordered/Reported per Month }\end{array}$ \\
\hline Family medicine & $24 / 78$ & 25 & 14 \\
Family medicine, sports medicine or emergency medicine & $6 / 78$ & 14 & 24 \\
Internal medicine & $9 / 26$ & 22 & 4 \\
Neurology & $18 / 32$ & 17 & 10 \\
Orthopedic surgery & $18 / 27$ & 17 & 26 \\
Neurosurgery & $7 / 10$ & 5 & 23 \\
PMR & $7 / 10$ & 22 & 19 \\
Radiologists (non-neuroradiology) & $17 / 36$ & 16 & 63 \\
Radiologists (neuroradiology) & $6 / 7$ & 14 & 125 \\
Radiology residents & $8 / 15$ & 3 & 12 \\
\hline
\end{tabular}

the Department of Medical Imaging of the London Health Sciences Centre during the past calendar year, along with their ordering frequency. We included on this list all pertinent specialty physicians: family medicine, neurology, neurosurgery, orthopedic surgery, PMR, general internal medicine, emergency medicine, and sports medicine (including family physicians with subspecialty practices). The number of referring family physicians was further limited to those ordering $\geq 8$ studies per year to achieve a manageable cohort by identifying those who regularly investigate spine complaints. A total of 183 clinicians were selected.

All practicing radiologists at the University of Western Ontario were categorized in our radiologists' group as either neuroradiologist or non-neuroradiologist. All radiology residents exposed to neuroradiology reporting were also incorporated into the subject sample, and their responses were grouped with the non-neuroradiologists.

\section{Research Design}

A survey was designed in 3 parts (On-line Appendix A). Part I obtained the subject's demographic information pertaining to specialty, years in practice, and the frequency with which clinicians requested or radiologists reported lumbar spine CT, MR imaging, and plain radiographs. Clinicians were subcategorized on the basis of their years of experience into junior staff ( $0-6$ years), experienced staff (7-15 years), and senior staff ( $>15$ years).

Part II included 3 sample case scenarios (cases 1-3) designed to reflect typical CT and MR imaging reports for patients with lower back pain. The first scenario was a CT scan with normal findings; the second was a CT scan with abnormal findings, in which the findings were pertinent to the patient's symptoms; and the third was an MR imaging study with abnormal findings with pertinent findings and an additional incidental but important finding of a renal lesion. Six reports were composed for each scenario. The content varied from limited (labeled A and B) to moderate (labeled C and D) to detailed (labeled $\mathrm{E}$ and $\mathrm{F}$ ). The format was either prose (A, C, and E) or itemized (B, D, and F).

The content varied as to the level of detail included in the report. Limited-content reports had only a basic description. Moderate content included the history, study technique, previous comparison, and a final impression. Detailed content reports had more description of the abnormalities in addition to pertinent negative and positive findings. The impression consisted of a comment on study quality, further recommendations for imaging follow-up and referral, and additional unrelated and yet potentially important findings. In this section, the subject was requested to rank their level of satisfaction with each of the reports above (A-F) for each scenario on a Likert scale, ranging from 1 (poor) to 5 (excellent), with 3 as the midpoint (satisfactory).
Part III of the study solicited opinions regarding components of each report, including the need for a comment on technique, study quality, and availability of previous examinations for comparison, in addition to level and type of description preferred and recommendation preferences.

Once approval from our institution's research ethics review board was obtained, surveys were mailed to all subjects, followed by reminder surveys to those who did not initially respond.

\section{Statistical Methods}

The collected surveys were analyzed by using the $\chi^{2}$ test to establish differences between clinicians and radiologists and 1-way analysis of variance (Tukey-Kramer method) to establish differences in content and style preferences within groups. Comparison was made between clinicians and radiologists; neuroradiologists and non-neuroradiologists; and among neurological specialists (neurologists and neurosurgeons), non-neurological specialists (orthopedic surgeons, PMR, general internists, emergency medicine physicians, and family physicians with subspecialty training in either emergency medicine or sports medicine), and nonspecialized family physicians. Correlation was made with the number of years in practice and the number of studies ordered to establish any trends related to experience.

\section{Results}

\section{Demographics}

Of the 183 clinicians surveyed, 89 responded ( $49 \%$ reply rate), while 31 of the 58 radiologists responded (53\% reply rate) (Table 1). Four clinicians declined to respond due to an inactive spine practice (retired, stroke-based practice) or lack of exposure to spine imaging. Two radiologists declined due to inexperience reporting spine studies. The number of years of experience ranged from none (newly certified physicians or residents) to 39 years for both groups.

\section{Report Satisfaction Level}

There was progressively increasing preference given to moderate and detailed reports by both physician groups, with no difference related to content or style formats (Table 2). Satisfaction level averages ranged from 2 to 4 on the Likert scale for sample reports A through $\mathrm{F}$ in each case scenario. Radiologists consistently disliked limited-content reports. Clinicians, on the other hand, demonstrated a slight preference for detailed content reports in either style format, though this difference was not statistically significant. 
Table 2: Clinicians' and radiologists' ranked satisfaction levels with different sample reports ${ }^{\mathrm{a}}$

\begin{tabular}{|c|c|c|c|c|c|}
\hline \multirow[b]{2}{*}{$\begin{array}{l}\text { Case } \\
\text { No. }\end{array}$} & \multirow[b]{2}{*}{ Style } & \multicolumn{2}{|c|}{ Clinicians } & \multicolumn{2}{|c|}{ Radiologists } \\
\hline & & Mean & $\begin{array}{l}\text { Level of } \\
\text { Correlation }\end{array}$ & Mean & $\begin{array}{l}\text { Level of } \\
\text { Correlation }\end{array}$ \\
\hline \multirow[t]{6}{*}{ Case 1} & A & 2.1 & $D$ & 2.0 & $B$ \\
\hline & B & 2.2 & D & 2.1 & B \\
\hline & C & 3.1 & C & 3.1 & A \\
\hline & D & 3.5 & $B, C$ & 3.4 & A \\
\hline & E & 4.0 & $A, B$ & 3.7 & A \\
\hline & $\mathrm{F}$ & 4.1 & $A$ & 3.4 & A \\
\hline \multirow[t]{6}{*}{ Case 2} & A & 2.4 & C & 2.5 & B \\
\hline & B & 2.5 & C & 2.3 & B \\
\hline & C & 3.5 & B & 3.6 & A \\
\hline & D & 4.0 & $A, B$ & 4.1 & A \\
\hline & $\mathrm{E}$ & 3.9 & $A, B$ & 3.4 & A \\
\hline & $\mathrm{F}$ & 4.1 & $A$ & 3.6 & A \\
\hline \multirow[t]{6}{*}{ Case 3} & A & 2.2 & C & 2.0 & B \\
\hline & B & 2.3 & C & 2.1 & B \\
\hline & C & 3.4 & B & 3.4 & A \\
\hline & D & 3.8 & $A, B$ & 3.7 & A \\
\hline & E & 3.9 & $A$ & 3.6 & A \\
\hline & $\mathrm{F}$ & 4.3 & A & 3.9 & A \\
\hline
\end{tabular}

${ }^{a} \mathrm{SD}=1$.

${ }^{\mathrm{b}}$ Levels not connected by the same letter are significantly different at $P<.01$.

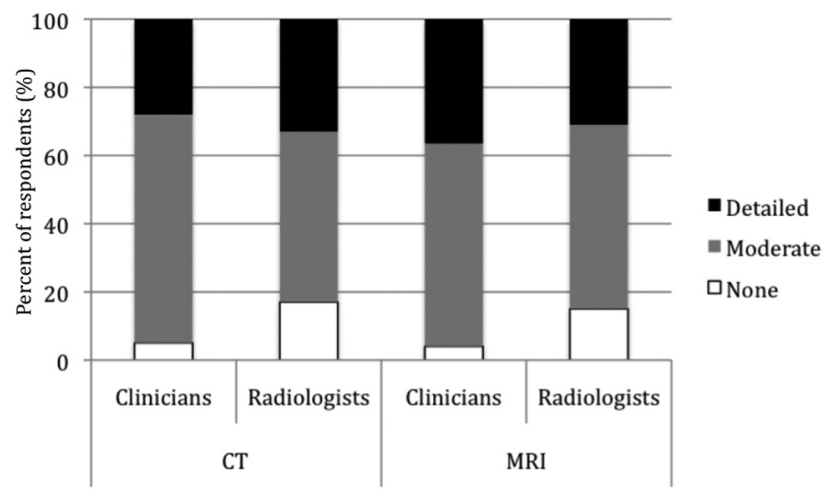

Fig 1. Clinicians' and radiologists' preferences for the level of study technique description reported in CT and MR imaging reports of the lumbar spine.

\section{Report Content}

Radiologists versus Clinicians. Most clinicians (88\%) and radiologists (75\%) agreed that the patient's history should be included in the report. They preferred moderate description of CT and MR imaging technique over none or detailed descriptions (Fig 1). There was disagreement between the 2 groups, however, regarding the quality of the CT scan being reported; radiologists (63\%) preferred to provide no comment about it, whereas clinicians $(83 \%)$ wanted to see it reported $(P<.001$, Fig 2). Some of the respondents (both clinicians and radiologists) deeming quality reporting to be unnecessary indicated that the scan quality should be reported only if it is poor or if the study should be repeated. More clinicians (89\%) than radiologists $(58 \%)$ thought that the quality of MR imaging should be described $(P<.001)$.

The absence of prior imaging was found to be relevant ( $80 \%$ of clinicians and $75 \%$ of radiologists), and comparison studies were also valued in the report by all participants (100\% of respondents).

Moderate-to-detailed description of normal CT findings was the preferred level for both radiologists and clinicians (Fig

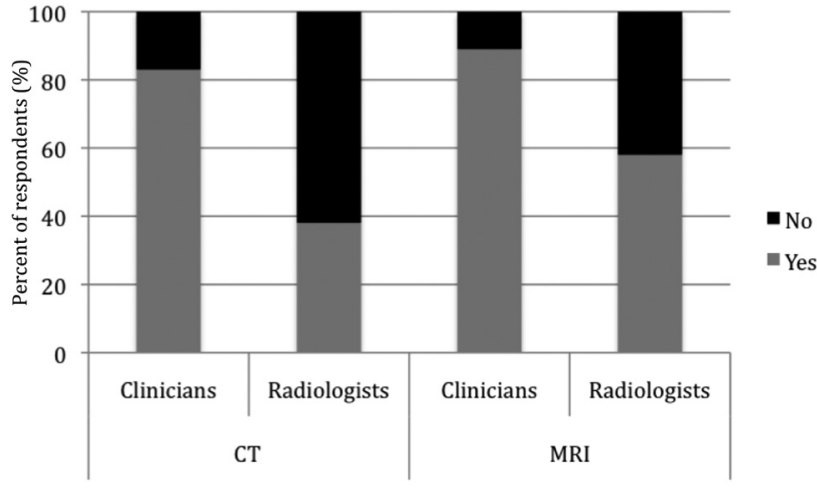

Fig 2. Clinicians' and radiologists' preferences regarding comment on CT and MR imaging study quality $(P<.001)$.

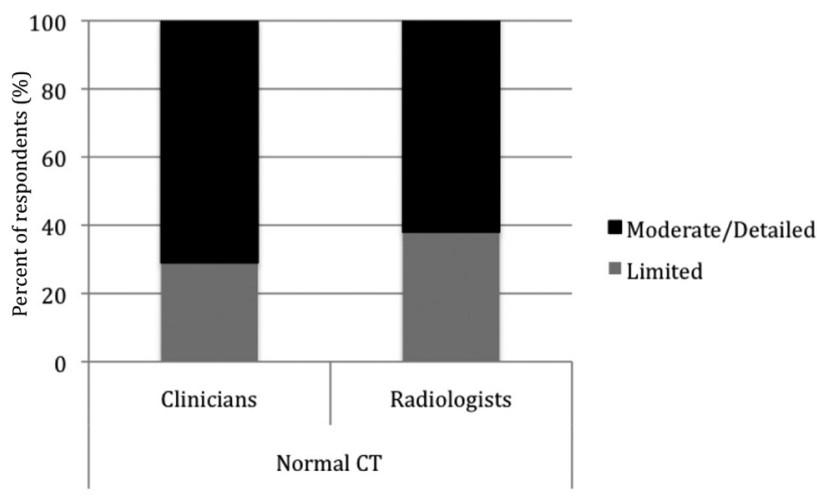

Fig 3. Preferred level of description of normal findings for a CT scan.

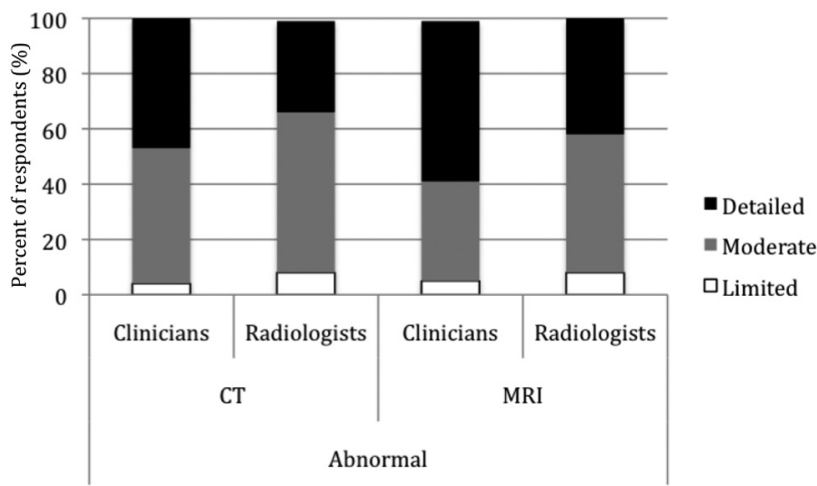

Fig 4. Preferred level of description of abnormal findings for a CT or MR imaging study. 3). An almost equal percentage of subjects preferred moderate and detailed CT descriptions when the study demonstrated pathology (Fig 4). Very few physicians chose limited description. Similar choices were made for the description of MR imaging findings, with a slight preference for additional detail by the clinicians. Qualitative descriptors were preferred by both groups for disk herniation on a CT scan with abnormal findings (56\% of clinicians and $64 \%$ of radiologists). Eight clinicians (9\%) and 1 radiologist (3\%) preferred both qualitative and quantitative descriptors and were excluded from the data analysis. When an incidental mass was suggested on the MR imaging, quantitative size descriptors were favored by both groups ( $61 \%$ of clinicians, $85 \%$ of radiologists, $P=.011$ ) over qualitative descriptors, while 10 clinicians requested both qualitative and quantitative descriptors and were excluded from data analysis. 


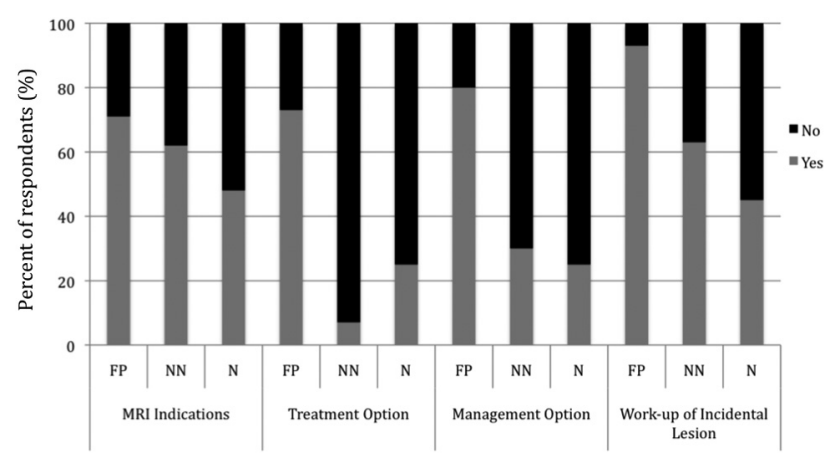

Fig 5. Family physicians' and non-neurological and neurological specialists' preferences for the radiologist's comments on MR imaging indications when the CT findings are normal $(P<.05)$ and recommendations for treatment (epidural steroid injection, $P<.001$ ), management options (surgical referral, $P<.01$ ), and the work-up of an incidental finding (renal mass, $P<.01$ )

When an abnormality was identified, pertinent negatives were deemed useful by both clinicians $(89 \%)$ and radiologists $(92 \%)$. Both groups also agreed that the radiologist's impression of normal ( $72 \%$ of clinicians and $71 \%$ of radiologists) or abnormal (96\% of clinicians and $92 \%$ of radiologists) findings on a study was valuable. Although clinicians wanted the radiologist to provide MR imaging indications when the CT findings were normal (59\%) or abnormal (82\%), most radiologists did not deem that necessary when the CT findings were normal $(58 \%)$ but were willing to provide it when they were abnormal (85\%). Clinicians (67\%) and radiologists (69\%) agreed that the recommendation of an epidural steroid injection for radiculopathy should not be mentioned. Radiologists (76\%) and clinicians (68\%) thought the recommendation for the work-up of an incidental renal mass was appropriate.

A slight majority of respondents disagreed with radiologists recommending a specialist referral for fracture management (55\% of clinicians and $54 \%$ of radiologists). Both physician groups were divided as to the value of recommending vertebroplasty for a lumbar fracture (51\% of clinicians and $62 \%$ of radiologists agreed to its inclusion). The ideal content of the radiology report of lumbar spine cross-sectional studies has been summarized (On-line Table).

Clinician Subspecialty Analysis. In addition to the differences established between clinicians and radiologists, further differences were demonstrated among clinician groups. When the CT study findings were normal, more neurological specialists (96\%) than non-neurological specialists (86\%) were interested in knowing the quality of the study, with family physicians being the least interested $(62 \%, P=.01)$. MR imaging quality was also valued more by the neurological specialists (100\%) than by the non-neurological specialists (89\%) and even more than by the family physicians $(80 \%, P=.02)$.

When further assessing the clinicians' preferences, we found that an increasing number of physicians wanted MR imaging indications to be reported when the CT findings were abnormal (75\% of neurological specialists, 78\% of non-neurological specialists, and $93 \%$ of family physicians) compared with when the study findings were normal (48\% of neurological specialists, $62 \%$ of non-neurological specialists, and $71 \%$ of family physicians, $P=.03$ ), with neurological specialists being the least likely to rely on this information (Fig 5). A significant difference was also established among clinicians, in which more family physicians (73\%) appreciated the recommendation for epidural steroid injection as a management option for radiculopathy while only a few specialists did (7\% of non-neurological and $25 \%$ of neurological specialists, $P<$ $.001)$. The same trend was demonstrated for the recommendation of specialist referral, which was appreciated by family physicians (80\%) more often than by the other groups (30\% of non-neurological and $25 \%$ of neurological specialists, $P<$ $.01)$. When an incidental renal mass was found, work-up recommendations were appreciated by most family physicians (93\%) and most of the specialists $(63 \%$ of non-neurological and $45 \%$ of neurological specialists, $P<.01$ ). The suggestion of vertebroplasty for a spinal fracture was appreciated by a slightly greater number of family physicians (67\%) than neurological specialists (55\%) and non-neurological specialists $(33 \%, P<.5)$.

Neuroradiologists versus Non-Neuroradiologists. Neuroradiologists favored a detailed $(60 \%)$ or moderate $(40 \%)$ description of CT study technique, while non-neuroradiologists preferred a moderate $(53 \%)$ over detailed $(26 \%)$ or limited description $(21 \%)$. A similar pattern was seen in MR imaging-technique description, in which most neuroradiologists (75\%) preferred detailed over moderate (25\%) description and non-neuroradiologists preferred moderate (59\%) over limited (18\%) or detailed (23\%) description. More neuroradiologists (80\%) than non-neuroradiologists (58\%) also believed in describing the CT findings in detail when they were normal. Neuroradiologists strongly believed that MR imaging indications should be given when the CT findings are abnormal (100\% agreed) and withheld when they are normal $(80 \%)$. Non-neuroradiologists were equally divided when the CT findings were normal (47\% for and 53\% against), but a larger number (82\%) were willing to provide these indications when the CT findings were abnormal.

MR imaging - quality description was valued by all neuroradiologists $(100 \%)$, while non-neuroradiologists were equally divided (50\%) as to whether this should be included in the report. Although clinicians and radiologists agreed that epidural steroid injections should not be recommended for radiculopathy, further analysis revealed that most neuroradiologists $(75 \%)$ deemed it valuable to provide this recommendation, a belief not shared by non-neuroradiologists (only $23 \%$ agreed). There were differences between groups as to the value of recommending specialist referral. Neuroradiologists $(75 \%)$ valued this idea, while non-neuroradiologists did not (59\%). The recommendation of vertebroplasty was also valued by all neuroradiologists, while most non-neuroradiologists did not $(73 \%, P<.1)$ value it.

\section{Impact of Physician Experience and Degree of Use on Preferences}

Qualitative description of a herniated disk was preferred by more junior $(80 \%)$ than experienced $(56 \%)$ or senior $(45 \%)$ staff $(P<.1)$. Junior staff $(100 \%)$ were also keener on having the CT scan quality included than the experienced or senior staff (78\% each group, $P<.1$ ). The need for work-up recommendations of the incidental finding increased with years of experience, with fewer junior staff (40\%) than experienced $(67 \%)$ and senior $(71 \%)$ staff relying on this recommendation $(P<.5)$. When the CT findings were abnormal, MR imaging 
indications were appreciated more by experienced (78\%) and senior $(90 \%)$ staff than their junior colleagues $(50 \%, P<.1)$.

The number of studies ordered by clinicians or reported by radiologists did not have a significant effect on the subjects' responses in the study.

\section{Additional Comments}

Family physicians provided comments encouraging recommendations pertaining to management options from radiologists. Neuroradiologists also indicated that indications for an MR imaging should be provided in a CT spine report, especially for family physicians. A few non-neuroradiologists also acknowledged the need to recommend additional management steps for family physicians, indicating that specialists may already have a plan of action based on the clinical scenario. Other radiologists, however, stated that clinical presentations have a weak association with the imaging findings; therefore, recommendations are inappropriate. Fear of the medicolegal consequences of not pursuing the recommendations also prompted certain clinicians to encourage suggestive phrases rather than strong recommendations so as to allow the clinician flexibility in their plan of action. In addition, an orthopedic surgeon stated that radiologists should not recommend vertebroplasties, believing that it is out of the radiologist's scope of practice. This study was performed before the publication of the article by Kallmes et $\mathrm{al}^{4}$ and Buchbinder et $\mathrm{al}^{5}$ reporting no significant benefit from vertebroplasty treatment of spinal fractures. Another clinician viewed the encounter as a pure consultation for imaging interpretation and answered that no mention of treatment or management should be included. Also, because both orthopedic surgeons and neurosurgeons may be capable of managing lower back pathology, a "surgical consultation" is preferred as a recommendation over a specific specialist's referral (such as "neurosurgeon referral").

A few non-neuroradiologists commented that the study quality should only be mentioned if it limited the diagnostic capabilities of the test.

\section{Discussion}

Given that the radiology report is the main means of communication between the radiologist and the referring physician and that communication errors are the fourth most frequent primary allegation against radiologists in the United States, it is no wonder that extensive work has been invested in establishing the characteristics of an ideal radiology report. ${ }^{6}$ This study aimed to identify clinician and radiologist preferences regarding the format and content of the lumbar spine crosssectional radiology report.

\section{Format}

With the advent of voice-recognition systems, many studies have embarked on quests to establish clinicians' preferences of itemized or prose reports. Establishing structured reports as a preferred style would justify the acquisition of voice-recognition systems and possibly enhance the completeness of reports. Structured reports could facilitate practice analysis, outcome measurements, and patient-population studies. ${ }^{7}$ Naik et $\mathrm{al}^{8}$ conducted a similar study in the field of body sonography, with a similar subject sample size, which demonstrated the clear preference of itemized reports over prose, especially as detail increased. A recent British study by Plumb et $\mathrm{al}^{9}$ of hospital clinicians also established a preference for tabulated reports over prose. The same author conducted a survey of general practitioners and identified similar results. ${ }^{10}$ Our study, however, demonstrated clear preference for more detailed reports in the setting of lumbar spine cross-sectional imaging, without significant preference for either prose or itemized formats, suggesting that completeness is preferred over report style.

Our findings support another study comparing the efficiency of information transfer by using free text and structured format, which found both to be equally efficient and accurate for transmitting content, also implying that a complete report is effective, regardless of its format. ${ }^{11}$ These findings may be explained by 2 factors. First, clinicians use prose style when they are reporting themselves and may be more familiar with this format in the radiology report. Second, our prose sample reports were highly structured, which may have rendered the prose and itemized samples more similar and, thus, dampened the differences between the subjects' preferences. In other words, more striking differences between prose and itemized reports may have been established if our prose reports had been free-flowing.

Clinicians' preferences have also been previously noted to change, depending on the clinical scenario in the setting of chest radiography. ${ }^{12}$ This change, however, was not clearly demonstrated in our study because there was no significant difference, despite case scenarios of varying complexity and different imaging techniques used. This finding may possibly be explained by the extent of detail that is seen in lumbar spine cross-sectional imaging compared with plain radiography. In addition, chest radiography is often used as a screening test (such as in the preoperative setting); therefore, a simple "normal" report is satisfactory, whereas lumbar spine cross-sectional imaging is often performed for a more definitive investigation of a specific clinical problem.

\section{Content}

Although it had been previously established that specialists prefer brevity and generalists prefer thorough reports, our study confirmed that a moderate-to-high level of detail is important to both groups. ${ }^{13}$ Clinicians and radiologists consistently disapproved of limited-content reports, even when the study findings were normal. This disapproval is in contrast to chest radiology reports, for example, in which "normal examination" was deemed acceptable. ${ }^{12}$ The literature has previously also established that both hospital clinicians and general practitioners prefer details and recommendations. ${ }^{10,13}$

\section{Differences}

It was previously established that most clinicians greatly value comments on the technical quality of the examination, especially if the examination is suboptimal. ${ }^{13}$ This may stem from the fact that a study may be of nondiagnostic or poor quality such that the findings are questionable or the diagnosis is missed (for example, due to motion artifacts or suboptimal contrast timing). Our study results indicate that this component of the radiology report is even more important for specialists (especially neurological specialists) than for family 
physicians. The inclusion of comparison studies is also always valuable to the referring physician. This may reveal to the clinician that the findings are chronic or even reveal previously unknown medical history. The documentation of study quality and comparison studies will, therefore, ultimately impact patient care.

We are not aware of any prior study that assessed the value of the radiologist's recommendations to the referring physician in the setting of lumbar spine imaging. Recommendations (for management or additional imaging, referral, and work-up of an incidental finding) were valued by family physicians more than by specialists. Among radiologists, neuroradiologists tended to include more recommendations that are valued by family physicians than non-neuroradiologists did. Thus, the referring clinician's area of practice should play a role in deciding how much detail or which recommendations should be included. If the specialty of practice is unknown, it is safer to include more detail and recommendations in the report for the general practitioner who may benefit from them. It would also behoove the non-neuroradiologist to become proficient in providing these recommendations appropriately.

Recommendations for a work-up of an incidental finding and the indications for MR imaging when the CT findings were abnormal were also valued by the more senior colleagues compared with junior physicians. This outcome may be due to senior physicians trusting the expertise of the radiologist to provide the most current standard of practice for further imaging. Conversely, the more junior physicians may be more aware of the current standards rendering these recommendations superfluous. A description of the CT scan quality was found to be very valuable to junior staff compared with more senior staff. It is possible that this finding is related to the development of trust and understanding between the referring physicians and the radiologists with time, such that the radiologist would only comment on the quality of the study if it was suboptimal or nondiagnostic. Although it may be impractical to always base the radiology report on the number of years of experience of the referring physician, it is safer to include these factors in the report for the potential benefit.

The radiology report is a legal document that may dictate the patient's fate in terms of future actions. As such, the choice of words is important in making recommendations. Several clinicians commented that recommending certain actions binds the clinician to follow such a course or become exposed to medicolegal risk. Suggestive phrases may, therefore, be favored when there is an array of management options available. Recommendations should be reserved for absolutely critical cases.

\section{Strengths and Weaknesses}

Our large subject population included physicians from both community and tertiary practices, thus encompassing a variety of opinions, experiences, and expectations. Despite the large number of respondents, rivaling larger center studies, an analysis of nonrespondents was not performed unless subjects volunteered reasons for opting out of the study. The number of specialists in both the clinicians' and radiologists' groups was low compared with the nonspecialists, potentially dampening differences between the 2 groups. With our small band of neuroradiologists, typical of most radiology groups, the power of our study to detect statistically significant relationships was diminished. A multicentered study could compensate for this. Future research in this area may explore the utility of standardized lexicon in the assessment of spinal disease.

\section{Conclusions}

While structured prose and itemized formats were both satisfactory, clinicians almost universally agreed that the ideal CT or MR imaging lumbar spine report should be comprehensive. History, technique, study quality, and the availability of prior studies for comparison were all important to clinicians. Family physicians wished for specific management recommendations from the radiologists. Specialists, however, preferred more latitude to formulate their own management decisions. There were differences within the radiology group as well: Non-neuroradiologists were unlikely to recommend spine interventions, while neuroradiologists were comfortable with this role. To optimize patient care, radiologists should be mindful of these preferences and consider tailoring their reports to their audiences.

\section{Acknowledgments}

We extend special thanks to Dr. Steven Karlik and Mr. JeanPierre Eskander for their assistance with the statistical analysis of our study.

\section{References}

1. Kushner DC, Lucey LL, for the American College of Radiology. Diagnostic radiology reporting and communication: the ACR guideline. Am Coll Radiol 2005;2:15-21

2. Hall FM. The radiology report of the future. Radiology 2009;251:313-16

3. Burns KEA, Duffett M, Kho ME, et al. A guide for the design and conduct of self-administered surveys of clinicians. CMAJ 2008;179:245-51

4. Kallmes DF, Comstock BA, Heagerty PJ, et al. A randomized trial of vertebroplasty for osteoporotic spinal fractures. N Engl J Med 2009;361:567-79

5. Buchbinder R, Osborne RH, Ebeling PR, et al. A randomized trial of vertebroplasty for painful osteoporotic vertebral fractures. N Engl J Med 2009;361: 557-68

6. Cascade PN, Berlin L. Malpractice issues in radiology: American College of Radiology standard for communication. AJR Am J Roentgenol 1999;173:1439-42

7. Morioka CA, Siha U, Taira R. Structured reporting in neuroradiology. Ann N Y Acad Sci 2002;980:259-66

8. Naik SS, Hanbidge A, Wilson SR. Radiology reports: examining radiologis and clinician preferences regarding style and content. AJR Am J Roentgenol 2001;176:591-98

9. Plumb AA, Grieve FM, Khan SH. Survey of hospital clinicians' preferences regarding the format of radiology reports. Clin Radiol 2009;64:386-94

10. Grieve FM, Plumb AA, Khan SH. Radiology reporting: a general practitioner's perspective. Br J Radiol 2010;83:17-22

11. Sistrom CL, Honeyman-Buck J. Free text versus structured format: information transfer efficiency of radiology reports. AJR Am J Roentgenol 2005; 185:804-12

12. McLoughlin RF, So CB, Gray RR, et al. Radiology reports: how much descriptive detail is enough? AJR Am J Roentgenol 1995;165:803-06

13. Lafortune M, Breton G, Baudouin JL. The radiological report: what is useful for the referring physician? J Can Assoc Radiol 1988;39:140-43 